Meta

Journal des traducteurs

Translators' Journal

\title{
Un vocabulaire juridique bilingue canadien
}

\section{Lucie Lauzière}

Volume 24, numéro 1, mars 1979

La traduction juridique

URI : https://id.erudit.org/iderudit/003422ar

DOI : https://doi.org/10.7202/003422ar

Aller au sommaire du numéro

Éditeur(s)

Les Presses de l'Université de Montréal

ISSN

0026-0452 (imprimé)

1492-1421 (numérique)

Découvrir la revue

Citer cet article

Lauzière, L. (1979). Un vocabulaire juridique bilingue canadien. Meta, 24(1),

109-114. https://doi.org/10.7202/003422ar d'utilisation que vous pouvez consulter en ligne.

https://apropos.erudit.org/fr/usagers/politique-dutilisation/ 


\section{Un vocabulaire juridique bilingue canadien}

\section{Introduction : le projet JURIVOC}

La recherche qui porte sur le projet JURIVOC à la Faculté de droit de l'Université d'Ottawa depuis plus de dix ans, a donné pour résultat le développement de techniques nouvelles dans le domaine de l'informatique juridique, un sous-domaine de la jurimétrie ${ }^{1}$. En combinant la linguistique juridique, le bilinguisme et la lexicographie automatisée, le projet de recherche a démontré qu'on pouvait créer un système de traitement de vocabulaire applicable à la compilation d'un dictionnaire juridique bilingue canadien ${ }^{2}$.

Dans un pays où coexistent deux systèmes juridiques et deux langues officielles, subsiste toujours un besoin grandissant d'études poussées sur le vocabulaire juridique afin de favoriser une plus grande communication entre les juristes des deux systèmes et afin de permettre une meilleure compréhension du droit aux non-juristes qui sont tout autant assujettis à la connaissance et à l'observation des lois. La lexicographie juridique assistée par ordinateur demande l'apport de plusieurs disciplines. JURIVOC est donc un centre d'études interdisciplinaires qui tient compte de la dualité des systèmes juridiques et de la dualité des langues dans l'ensemble de son système de traitement du vocabulaire. Les deux systèmes juridiques ne sont pas complètement étanches au Canada. Le bilinguisme de la Common Law et du droit civil, tant dans l'enseignement et la pratique du droit que dans la rédaction des lois, favorise une interaction constante entre les membres des deux systèmes juridiques.

En matière de droit, on insiste sur l'importance de la précision du vocabulaire. La rédaction de textes législatifs bilingues, la traduction des lois et règlements déjà existants, la traduction des arrêts et ordonnances sont autant de raisons qui commandent un grand besoin d'uniformisation dans l'utilisation du vocabulaire juridique. La création d'un vocabulaire juridique bilingue canadien qui tend à ordonner l'usage des termes, surtout dans ce domaine où les sources

1. La jurimétrie se définit comme l'investigation scientifique des problèmes de la science juridique. Loevinger, « Jurimetrics - the next step forward 》, 33 Minnesota Law Review, 1949, p. 455.

2. L'ouvrage qui sert de point de départ à l'article est une publication du projet JURIVOC : Lexicographie, bilinguisme juridique et ordinateur, Viateur Bergeron, David C. Burke, Jeanne Leclerc-Houde, Michael Mepham, Roland Serrat, Les Editions de 1'Université d'Ottawa, 1976. 
documentaires sont abondantes, s'avère utile aux légistes, aux traducteurs-juristes et à tous ceux qui doivent lire et interpréter quotidiennement des textes juridiques. Ceux-ci auront en main au départ un outil de travail commun qui contribue tant à l'uniformisation de la terminologie à l'intérieur de chaque système juridique qu'à l'harmonisation des concepts utilisés dans les deux systèmes juridiques au Canada qui, rappelons-le, sont aussi les deux principaux systèmes juridiques du monde. Il n'appartient pas aux légistes ni aux traducteurs-juristes de décider de l'utilisation du vocabulaire juridique. Dans notre système, le législateur et, jusqu'à une certaine mesure, les tribunaux, décident du sens à donner aux mots des textes de loi. Ceux-ci doivent donc être rédigés en accord avec le vocabulaire imposé par le législateur ou les tribunaux. Il est donc important de mettre entre les mains de tous ceux qui doivent rédiger des textes de loi des instruments de travail qui leur permettent de le faire en respectant ce vocabulaire imposé.

\section{La linguistique automatique}

La création d'un dictionnaire juridique nécessaire comme étape préalable à la compilation de plusieurs lexiques juridiques et bilingues qui inventorient tout le vocabulaire juridique bilingue canadien. La conception d'un système de classification (thésaurus juridique) pour diviser le droit en domaines et sousdomaines et les relier entre eux dans un ensemble hiérarchique nous aide à comprendre le droit et à déterminer le domaine précis de chaque lexique juridique. Avec l'élaboration de la structure de classification nous apparait évidente la division des sources du vocabulaire pour les lexiques.

La plupart des sources sûres pour l'étude du vocabulaire juridique sont des textes de loi traduits. Les traductions officielles, c'est-à-dire sanctionnées par le Parlement, reçoivent un caractère d'égale valeur à la version originale d'un texte de loi $^{3}$ et rappellent de ce fait leur qualité irréprochable. La compilation de lexiques juridiques bilingues à partir des traductions qui existent déjà a l'avantage de nous procurer comme base de données terminologiques le travail et l'effort individuels de chaque traducteur-juriste tels que reflétés dans les lois. En somme, il y a beaucoup plus de bonnes traductions que de mauvaises dans nos lois actuelles. Ainsi le caractère officiel des deux versions d'un texte de loi d'une part et la mise en garde contre les mauvaises traductions d'autre part nous assurent des sources authentiques et la qualité scientifique de nos recherches.

Le dépouillement lexical des lois se fait par une équipe de juristes qui identifient les termes juridiques lors d'une analyse textuelle, un peu à la façon du linguiste ou de l'ethnologue qui fait une enquête sur le terrain, et tout le vocabulaire recueilli est alors emmagasiné dans un ordinateur. L'entrée des données sur ordinateur facilite par la suite la production automatique de listes alphabétiques bilingues à partir desquelles se fera l'étude des utilisations des termes individuels et de leurs correspondants. L'ordinateur compile et trie à

3. Art. 133 de l'Acte de l'Amérique du Nord britannique et art. 8(1) de la Loi sur les langues officielles, ch. 0-2, S.R.C. 1970. 
notre avantage un nombre très élevé de données et nous amène rapidement au stade de l'analyse terminologique.

\section{L'aspect bilingue et l'aspect bi-juridique}

La terminologie juridique se distingue des autres champs terminologiques par le fait qu'elle nous force à découper la réalité en tenant compte à la fois de l'aspect linguistique et de l'aspect juridique d'un terme ou d'un concept donné. Ainsi, pour assurer l'équivalence exacte d'une terminologie juridique bilingue, il faut s'assurer non seulement de la correspondance des deux langues mais aussi de la correspondance des deux systèmes juridiques auxquels les termes étudiés appartiennent respectivement.

Les entrées d'un lexique tirées des deux versions anglaise et française des textes de loi doivent se fonder sur une considération générale d'un nombre très large de contextes tirés de ces textes avant de passer au choix du meilleur correspondant ou du plus valable pour un terme ou une expression donnés. D'autre part, il faut délimiter et définir les concepts qui permettent leur expression bilingue. Il n'existe pas de vocabulaire scientifique commun à tous les systèmes juridiques du monde entier. Existe-t-il des concepts de droit communs à tous ces systèmes? Contrairement aux sciences exactes qui se rapportent à des procédés ou à des principes absolus et reconnus universellement, le droit se fonde sur des relations très nuancées. Le vocabulaire juridique possède une valeur variable et relative au système juridique qui l'élabore. Ainsi la traduction juridique appelle non seulement l'identité des termes linguistiques correspondants mais aussi l'identité des concepts juridiques. L'ensemble de la Common Law et du droit civil et les diverses expressions linguistiques qui s'y rattachent forment un tout dynamique facilement comparé et compris à l'aide d'un thésaurus juridique, c'est-à-dire à l'aide d'une structure hiérarchique du vocabulaire bi-juridique établissant des classes où se retrouvent les institutions juridiques similaires. Ainsi la conception d'un thésaurus met en relief la transparence des deux systèmes juridiques et nous aide à prendre le recul nécessaire par rapport aux termes à étudier.

\section{Le mortgage et l'hypothèque : un exemple classique}

L'absence de vocabulaire commun aux différents systèmes juridiques du monde présente aux terminologues un sujet plein d'embûches. Les deux systèmes juridiques en application au Canada se différencient par la nature des institutions sur lesquelles ils se fondent et par leur désignation linguistique. Une première observation sur la législation canadienne nous fait découvrir que les législations provinciales bilingues ne se rapportent toujours qu'à un seul système juridique (le droit civil au Québec et la Common Law dans toutes les autres provinces) alors que la législation fédérale bilingue doit rendre compte des deux systèmes juridiques à la fois. Comment peut-on assurer la parfaite équivalence conceptuelle et la bonne désignation linguistique d'un terme en passant d'un système juridique à un autre ? Plus concrètement, comment est-ce qu'on traduira le terme «hypothèque » qui fait partie de notre droit civil ? En faisant un dé- 
pouillement rapide des textes de loi sur la question (le Code civil de la province de Québec, le Code criminel du Canada, les Revised Statutes of New Brunswick), le traducteur-juriste aura le choix suivant :

a) dans la version anglaise du Code civil, l'on retrouve sans exception le terme « hypothec » pour traduire « hypothèque ».

Art. 2017 C.C.

L'hypothèque est indivisible et subsiste en entier sur tous les immeubles qui y sont affectés,...

Hypothec is indivisible and subsists in entirely upon all the immoveables made liable,...

b) dans les statuts du Nouveau-Brunswick, le terme «mortgage » est rendu par « hypothèque ».

Art. 15 Bills of Sale Act, chap. B-3, R.S.N.B. 1973

A sale or mortgage or a bill of sale that under this Act is void or has ceased to be valid as against a creditor or purchaser or mortgagee is not, by reason of the fact that the grantee has subsequently taken possession of the chattels sold or mortgaged,...
Une vente, une hypothèque ou un acte de vente qui, par l'application de la présente loi, est nul ou cesse d'être opposable à un créancier ou à un acheteur ou créancier hypothécaire ne redevient pas, en raison du fait que l'acquéreur a par la suite pris possession des biens personnels vendus ou hypothéqués,...

c) dans le Code criminel, le terme «mortgage» est toujours traduit par * hypothèque », sauf lorsqu'il est employé conjointement avec le terme «hypothec »; celui-ci est alors traduit par \& hypothèque » et *mortgage * est traduit par \& mortgage 4 ».

Art. 343 C. cr.

Every one who... is served with a written demand or an abstract of title by or on behalf of the purchaser or mortgagee before the completion of the purchase or mortgage...

Art. 345 C. cr.

Every one who, knowing of an unregistered prior sale or of an existing unregistered grant, mortgage, hypothec, privilege... quiconque... ayant reçu formellement une demande écrite de fournir un extrait de titre par l'acquéreur ou par le créancier hypothécaire, ou au nom de l'acquéreur ou du créancier hypothécaire, avant que l'achat ou l'hypothèque soit complété...

quiconque, étant au fait d'une vente antérieure non enregistrée ou de quelque concession, mortgage, hypothèque, privilège...

4. Criminal Law - Droit criminel, English-French, (Specimen) Donat Pharand et Viateur Bergeron, JURIVOC, Université d'Ottawa, novembre 1970, non publié. 
Cet inventaire peut être rapidement repéré par ordinateur. On adresse une requête à l'ordinateur pour relever tous les contextes dans lesquels un mot clé, \& hypothèque \& par exemple, est employé dans le Code criminel. L'ordinateur fait une lecture rapide de toutes les pages du Code criminel déjà emmagasinées dans sa mémoire et dresse aussitôt une liste de tous les contextes où se trouve le mot « hypothèque ».

A priori, les deux termes \&hypothec » et « mortgage 》 semblent interchangeables. Mais il faut faire un pas de plus et procéder à l'étude du mot en situation, c'est-à-dire en tenant compte du système de référence d'où il tire sa signification. En droit civil, l'hypothèque est un droit réel sur un bien immeuble garantissant le paiement d'une dette ; elle n'opère pas un transfert de propriété mais permet à celui qui la détient de faire vendre l'immeuble en justice et d'être colloqué par la suite sur le prix de la vente. Au Québec, seuls les immeubles peuvent être hypothéqués, sauf rares exceptions ${ }^{5}$. Dans la Common Law, le 《 mortgage » est défini comme « the pledging of property to a creditor as security for the payment of a debt ${ }^{B} \gg$. Il s'applique tant aux biens meubles qu'aux biens immeubles et transfère au créancier de la dette le droit de propriété. Il y a donc des différences fondamentales entre les deux institutions.

Dans la version anglaise du Code civil, l'emploi du terme « hypothec traduit avec justesse la désignation linguistique et l'équivalence notionnelle du terme "hypothèque ». Les deux se rattachent uniquement au droit civil québécois et l'on évite ainsi toute confusion entre des institutions juridiques différentes.

Que penser du terme «mortgage » de la Common Law rendu en français par « hypothèque \&? L'hypothèque et le mortgage ont des points en commun, mais en réalité celui-ci s'apparente plus à la vente à réméré en droit civil qu'à l'hypothèque ?. De toute façon, la comparabilité de ces termes laisse à désirer et il vaut mieux ne pas forcer le rapprochement d'institutions juridiques n'ayant pas la même structure ni la même fonction. Le mieux que l'on puisse suggérer, c'est peut-être de renoncer à traduire et de conserver le terme \&mortgage » dans la version française d'un texte de loi. Cette solution, au moins, offre l'avantage d'assurer le parallélisme juridique et linguistique de l'institution désignée dans les deux versions législatives et, encore une fois, d'éviter toute confusion entre une institution de droit civil et une autre de la Common Law. Par ailleurs, quand on traduit le terme «mortgage » par «hypothèque », on ne traduit rien du tout! Dans ce cas, la transposition juridique que doit opérer la traduction est fallacieuse. Le raisonnement risque d'être erroné si la précision des termes et la stabilité des rapports reliant les termes aux concepts juridiques ne sont pas assurées ${ }^{8}$. Cette remarque vaut aussi pour le cas de l'article $343 \mathrm{C}$. cr. dont

5. Art. 2016 du Code civil, art. 22 et s. de la Loi des pouvoirs spéciaux des corporations, ch. 275, S.R.Q. 1964 et amendements ; art. 165 (c) (d) de la Loi des compagnies du Québec, ch. 271 , S.R.Q. 1964 et amendements.

6. Criminal Law Droit criminel, English-French, (Specimen) Donat Pharand et Viateur Bergeron, JURIVOC, Université d'Ottawa, novembre 1970, non publié.

7. Id.; ibid.

8. Traité de droit comparé, tome II, La méthode comparative, Léontin-Jean Constantinesco, Librairie de Droit et de Jurisprudence, Paris, 1974, p. 147. \& La traduction terminologique consiste a superposer, par l'entremise des deux termes concordants, appartenant à deux terminologies juridiques différentes, deux concepts ou notions juridiques identiques, équivalents ou correspondants, appartenant à deux droits différents. * 
l'application territoriale s'étend à toutes les provinces du Canada. La rédaction de cet article soulève une divergence entre ses deux versions linguistiques. En somme, on pourrait dire, si ce n'était de l'article $8(2) \mathrm{c}$ ) de la Loi sur les langues officielles ${ }^{\circ}$, qu'une large quantité de biens meubles est soumise à 1'application de la version anglaise de l'art. $343 \mathrm{C}$. cr. alors qu'elle est exclue de l'application de la version française du même article, par l'emploi du terme "hypothèque » qui, on l'a déjà souligné, sauf exceptions, ne se rapporte qu'aux immeubles. Cette controverse peut facilement être écartée en faveur d'un meilleur équilibre entre les deux versions en adoptant le principe de rédaction de l'article $345 \mathrm{C}$. cr., soit l'inclusion des deux institutions juridiques dans chaque version officielle. Ainsi, dans la version française, il sera impossible d'attacher au terme «mortgage » une définition autre que celle que lui donne la Common Law et dans la version anglaise, le terme "hypothec» aura un sens identique à celui que lui donne l'article 2016 du Code civil. Le créancier hypothécaire (hypothecary creditor) se distinguerait du créancier sur mortgage (mortgagee) et le débiteur hypothécaire (hypothecary debtor) du débiteur sur mortgage (mortgagor) ${ }^{10}$.

L'article $8(2)$ c) de la Loi sur les langues officielles pourrait alors être réformé en ce sens. L'exemple de l'article $345 \mathrm{C}$. cr. devrait être suivi dans tous les autres articles du Code criminel où l'on fait mention de ces concepts, de façon à ce que chaque version couvre à la fois l'hypothèque du droit civil et le mortgage de la Common Law comme si l'on procédait à deux rédactions parallèles et à deux traductions indépendantes, qu'on amalgame par la suite dans un texte législatif final.

LUCIE LAUZIÈre

9. Art. $8(2)$ c) de la Loi sur les langues officielles, ch. 0-2, S.R.C. 1970. « Lorsque l'expression d'un concept ou d'une chose, dans l'une des versions du texte législatif, est incompatible avec le système juridique ou les institutions d'un lieu du Canada où l'on veut que ce texte s'applique mais que son expression dans l'autre version du texte est compatible avec ce système ou ces institutions, une mention du concept ou de la chose dans le texte sera, dans la mesure où ce texte s'applique à ce lieu du Canada, interprétée comme une mention du concept ou de la chose, exprimée dans la version qui est compatible avec ce système ou ces institutions $\gg$, demande aux juristes anglophones de formation civiliste d'interpréter le terme «mortgage» de la version anglaise de l'art. 343 C.cr. comme synonyme de «hypothèque » et aux juristes francophones de la Common Law d'interpréter le terme « hypothèque » comme synonyme de « mortgage». A quoi sert la traduction alors ?

10. Il ne s'agit pas de savoir si le législateur a voulu traiter la province de droit civil et les provinces de Common Law sur un pied différent en matière de titre de propriété, mais plutôt de voir à donner tout l'effet au principe que les versions des deux langues officielles font pareillement autorité. La Cour d'Appel de l'Ontario a d'ailleurs déjà tranché le litige affirmativement sur ce premier point dans The King v. McDevitt (1910) 22 O.L.R. 490, en soutenant que le terme «privilège» employé à l'article 345 C.cr. est un mot technique de la loi civile du Québec et que dans ce sens il ne s'applique qu'à cette province. En suivant cette décision, l'on peut induire que le mortgage n'aura d'application que dans les provinces de Common Law et que l'hypothèque ne s'appliquera qu'au Québec. 